\title{
Design and optimization of multi user OFDM orthogonal chaotic vector shift keying communication system
}

\author{
Ansam M. Abed, Fadhil S. Hasan
}

Electrical Engineering Department, Al-Mustansiriya University, Baghdad, Iraq

\begin{tabular}{l} 
Article Info \\
\hline Article history: \\
Received Sep 26, 2020 \\
Revised Nov 22, 2020 \\
Accepted Dec 16, 2020 \\
\hline
\end{tabular}

Keywords:

Multiuser OFDM-DCSK

OCVSK

Power allocation strategies

\begin{abstract}
This paper study and present, power allocation strategy on sub-carriers of multiuser OFDM employed for orthogonal chaotic vector shift keying (MU OFDM-OCVSK) over multipath frequency selective fading channels. firstly, the MU OFDM-OCVSK system is modeled with power allocated on reference and information bearing subcarriers. Then, the computed bit error rate equation of the power allocation MU OFDM-OCVSK system is derived. The optimal power allocation strategy on subcarriers is obtained using convex optimization. Finally, compared with the traditional MU OFDMDCSK and MU OFDM-OCVSK without power allocation system the proposed system can achieve an excellent BER performance under multipath Rayleigh fading channels. Numerical and Simulation results emphasize the remarkable features of the proposed optimal power allocation strategy.
\end{abstract}

This is an open access article under the CC BY-SA license.

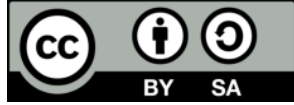

\section{Corresponding Author:}

Ansam M. Abed

Electrical Engineering Department

Al-Mustansiriya University, Baghdad, Iraq

Email: ama_ansam@yahoo.com

\section{INTRODUCTION}

Differential chaos shift keying (DCSK) is an attractive technique that preferred among a number of chaotic modulation schemes due to its robustness to multipath fading environments and non-coherent ability of implementation and simple transceiver requirement. In DCSK system every bit duration is split into two equal slots and a reference chaotic sequence is transmitted within the first time duration. According to the value of the bit to be sent, the reference signal is either copied or inverted and sent within the second time slot [1-3]. Many DCSK systems have been studied to evaluate its performance under different scenarios. In fact, the primary defect of the DCSK is that half of the bit's time slot in this system is employed for bearing reference signals so it is spent sending no-data. This can be considered as a significant data rate reducer and energy-inefficient for transmission [4-10].

The high efficiency HE-DCSK has been proposed in [4], as a new non-coherent system to overcome this defect and to improve the DCSK system performance partially also M-DCSK differentially DDCSK and reference modulated RM-DCSK, are new systems proposed to improve DCSK system, but they add complexity to the system and they are difficult to implement [5-7]. A code-shifted DCSK (CS-DCSK) system is presented in which a Walsh code sequences are used to separate the reference and information sequences instead of time delay lines. Then the modified version of this system is offered in [8-9] in which different chaotic sequences displaced the Walsh codes but, at the receiver in these two approaches it demands the generation of Walsh or chaotic codes that impacts the non-coherent behavior of the DCSK communication system. A new DCSK based on orthogonal chaotic vectors is presented in [10], in which Gram-Schmidt process is applied to produce orthogonal chaotic signals. To increase the data rate and improve the spectral 
efficiencies and total system energy the authors in [11] presented a new DCSK scheme that combines code index modulation with DCSK referred to as CIM-DCSK.

A multicarrier DCSK (MC-DCSK) system have proposed in [12, 13], the system was developed to support multi-access ability that was enhanced the conventional of the DCSK system. This system provides better data rates, enhances the energy efficiency, however it spends bandwidth and requires using parallel matched filters. In other words, orthogonal frequency division multiplexing (OFDM) is the more dominant multicarrier for discrete signals in nowadays technology [14, 15]. OFDM-DCSK system is suggested to combine the advantages of OFDM and DCSK system, decrease the complexity of the multicarrier DCSK system and offer better spectral efficiency compared to conventional DCSK systems [16-18].

In [19] the authors presented the multi-user OFDM-DCSK to support multi-user communication. Although this system enhances the spectral and energy efficiency, it increases multiple access interference (MAI). The nonlinear multi-user OFDM-DCSK communication system is studied and presented in [20] which considering the nonlinearity of power amplifiers distortion that degrades the BER performance of the system. Many power allocation schemes were introduced to enhance the overall performance and achieve different purposes such as maximizing the throughput, and ensure the Quality of Service demand for the primary and secondary users [21-23]. To enhance the performance in Multi Carrier DCSK and multi carrier QCSK systems over AWGN channel, power allocated to the references and information-bearing sequences are proposed as in [24, 25], although the poor channel conditions have not been taken into consideration for the two systems. Power optimization in the multi-user OFDM based DCSK over multipath fading channels have been proposed in [26].

In this paper, a MU OFDM-OCVSK system with subcarriers power allocation is proposed. The optimization trouble of subcarriers power allocation is determined using convex optimization and the BER expression of MU OFDM-OCVSK is derived with subcarriers power allocation based on the proposed model. The performance comparison between conventional MU OFDM-DCSK, a multi-user OFDM employed for orthogonal chaotic vector shift keying (MU-OFDM-OCVSK) and optimal power allocated counterpart system are also introduced.

The rest of the paper is arranged as the following, in section 2, the subcarriers power allocated MUOFDM-OCVSK system model is illustrated. The system performance is investigated, and analytical BER is derived in section 3. Section 4 illustrates the simulation results with the discussions. The conclusions are presented is depicted in section 5 .

\section{SYSTEM MODEL}

The structure of the transmitter for the $\mathrm{u}^{\text {th }}$ user in MU-OFDM- OCVSK system with power allocation strategy on subcarriers for $\mathrm{Np}=1$ is shown in Figure 1 .

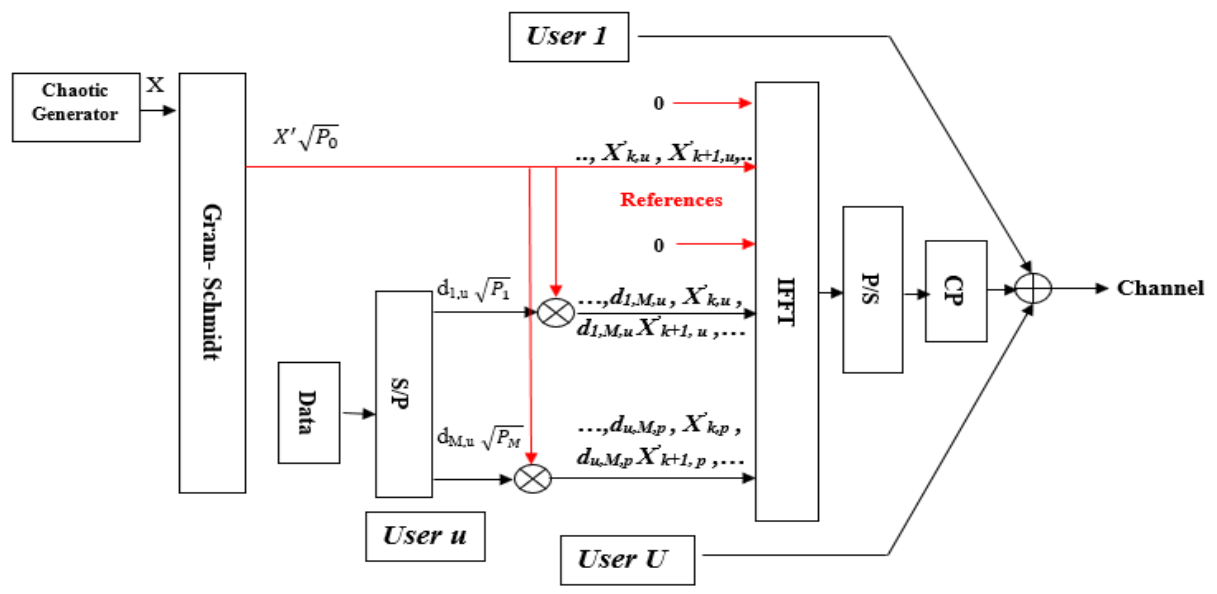

Figure 1. The power allocated MU OFDM-OCVSK transmitter

For the MU-OFDM-OCVSK, the reference vectors of various users $x_{u}(t)$ are sent over predefined frequencies that are used for both as reference signals and code for spreading the data bits M of user $u$ while $M$ bit stream $\mathrm{s}_{\mathrm{i}}=\{+1,-1\}$ are multiplied by the orthogonal chaotic spreading code $x_{u}(t)$ in time domain. 


$$
x_{u}(t)=\sum_{k=1}^{\beta} x_{u, k} g\left(t-k T_{c}\right)
$$

where $g(t)$ is a rectangular shaping filter, $\beta$ is the spreading factor and $T_{c}$ is the chip duration and its assumed that $T_{c}=1$ for simplicity. So, the transmitted signal of the $\mathrm{u}^{\text {th }}$ user of OFDM-OCVSK system is given by:

$$
S_{u}(t)=\sum_{k=1}^{\beta} x_{k, u} e^{2 \pi j f_{o}\left(t-k T_{c}\right)} g\left(t-k T_{c}\right)+\sum_{i=U+1}^{U+M} \sum_{k=1}^{\beta} x_{k, u} d_{i, u} e^{2 \pi j f_{i}\left(t-k T_{c}\right)} g\left(t-k T_{c}\right)
$$

where $S_{u}(t)$ is the OFDM symbol transmitted by user $\mathrm{u}, \mathrm{u} \in\{1, \ldots, \mathrm{U}\}$ and $f_{o}$ is its $\mathrm{u}^{\text {th }}$ private frequency used for transmitting the chaotic reference signal $x_{k, u}$, and $f_{i}$ is the $\mathrm{i}^{\text {th }}$ public sharing frequency.

Inspired by the comb-type pattern in OFDM system The frame for the proposed MU OFDM-DCSK consist of $U$ users each user has a number $N_{p}$ of predefined subcarrier used for sending their reference signals with total number of $N_{p} U$ subcarriers out of total $N$ subcarriers and the rest $N_{s}=N-N_{p} U$ subcarriers are shared by all users used for transmitting their data. The chaotic generator generates carriers using a secondorder Chebyshev polynomial function (CPF) [19]:

$$
x_{k+1}=1-2 x_{k}^{2}
$$

Figure 2 illustrates MU-OFDM-OCVSK structure at receiver side. The receiver structure allowing us to carry out our proposed power allocation technique without providing the frequency diversity and leads to a correct detection using a simple FFT operation replace the parallel matched filters used in [11] to reduce complexity which is one of the objectives of the design.

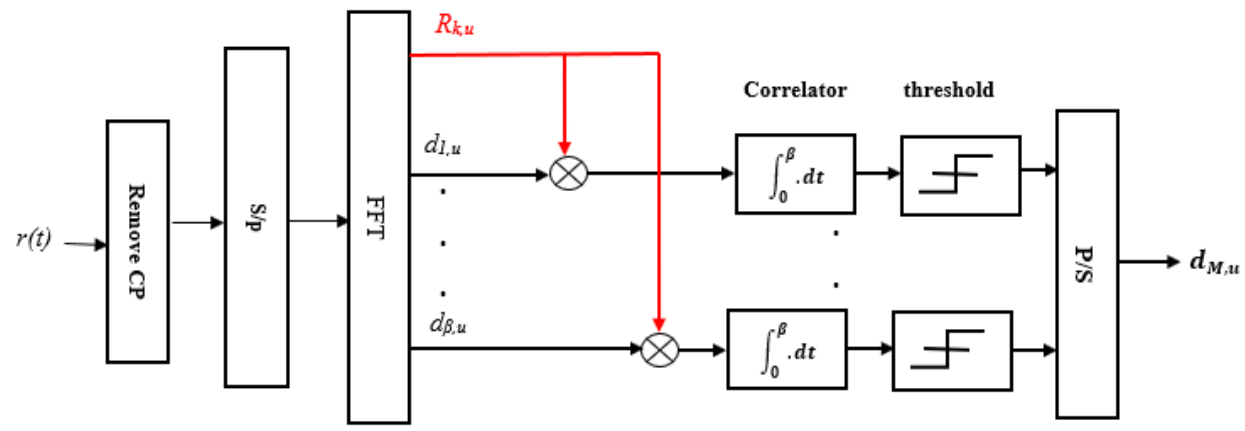

Figure 2. Block diagram for $U^{\text {th }}$ user of MU OFDM-OCVSK receiver

\section{BER DERIVATION AND POWER OTIMIZATION ON SUBCARRIERS}

In this section, the analytic BER of MU-OFDM-OCVSK with power allocation strategy on subcarriers is investigated. Define $\mathbf{P}=\left[P_{0}, P_{1}, \ldots, P_{M}\right]$ as the power vector of the reference and data bearing sequences for each user of OFDM-OCVSK system. In (2) can be rewritten as:

$$
S_{u}(t)=\sqrt{P_{0}} \sum_{k=1}^{\beta} x_{k, u} e^{2 \pi j f_{o}\left(t-k T_{c}\right)} g\left(t-k T_{c}\right)+\sum_{i=1+U}^{U+M} \sum_{k=1}^{\beta} \sqrt{P_{i}} x_{k, u} d_{i, u} e^{2 \pi j f_{i}\left(t-k T_{c}\right)} g\left(t-k T_{c}\right)
$$

The bit energy required to transmit one OFDM-DCSK bit can be written as [16]:

$$
E_{B}=E_{\text {data }}+\frac{N_{p} E_{r e f}}{M}=\frac{N_{p}+M}{M} \sum_{k=1}^{\beta} x_{k}^{2}
$$

By applying power allocation strategy on subcarriers $E_{B}$ will be,

$$
E_{B}=\frac{1}{M} \sum_{m=1}^{M+N_{p}} P_{m} \sum_{k=1}^{\beta} x_{k}^{2}
$$

At the receiver side, the data of each user are detected by calculating the sign of the following decision variable:

$$
D_{i, u}=\operatorname{Re}\left\{\sum_{k=1}^{\beta} Y\left(k, i, f_{s}\right) \cdot R\left(k, f_{P}\right)^{*}\right\}, i=1, \ldots, M
$$


where $R e\{$.$\} is the real part of the signal, and R\left(k, f_{P}\right)^{*}$ is the conjugate of the reference sequence $R\left(k, f_{P}\right)$. And,

$$
\begin{aligned}
& Y\left(k, i, f_{s}\right)=\sum_{u=1}^{U} \sqrt{P_{i}} d_{i, u} x_{k, u} \alpha_{u, l}+N_{s} \\
& R\left(k, f_{P}\right)=\sqrt{P_{0}} x_{k, u} \alpha_{u, l}+N_{P}
\end{aligned}
$$

where $N_{s}, N_{P}$ and $\alpha_{u, l}$ are independent zero mean AWGN and the channel coefficient response respectively. The decision variable $D_{i, u}$ may be expanded as:

$$
\begin{aligned}
& D_{i, u}=\mathbb{R}\{\underbrace{\sqrt{P_{0} P_{i}} d_{i, u} \sum_{l=1}^{L_{p}} \sum_{k=1}^{\beta}\left|\alpha_{u, l}\right|^{2} x_{k, u}^{\prime}{ }^{2}}_{A}+ \\
& \underbrace{\sum_{l=1}^{L_{p}} \sum_{k=1}^{\beta} x_{k, u}^{\prime}\left(\sqrt{P_{i}} d_{i, u} \alpha_{u, l} N_{P}^{*}+\sqrt{P_{0}} \alpha_{u, l}^{*} N_{S}\right)}_{D}+\underbrace{\sum_{k=1}^{\beta} N_{S} N_{P}^{*}}_{B}+ \\
& \underbrace{\sum_{r=1}^{U} \sum_{l^{\prime}=1}^{L_{r}} \sum_{k=1}^{\beta} \sqrt{P_{i}} \alpha_{r, l^{\prime}} d_{i, r} x_{k, r} N_{P}^{*}}_{E}+ \\
& \underbrace{\sum_{l=1}^{L_{u}} \sum_{\substack{r=1 \\
r \neq u}}^{U} \sum_{l^{\prime}=1}^{L_{r}} \sum_{k=1}^{\beta} \sqrt{P_{0} P_{i}} \alpha_{u, l}^{*} \alpha_{r, l^{\prime}} d_{i, r} x_{k, u}^{\prime} x_{k, r}}_{D}\}
\end{aligned}
$$

The first expression $\boldsymbol{A}$ represent the desired signal component while the expressions $\boldsymbol{B}$ and $\boldsymbol{C}$ represent the interference caused by AWGN. The expressions $\boldsymbol{D}$ and $\boldsymbol{E}$ are related to the multiple access interference (MAI) introduced in the decision variable. The orthogonality of the chaotic vectors makes $x_{k, u}^{\prime} x_{k, r}^{\prime}=0$, hence term $E$ equals zero. $L_{u}$ and $\alpha_{u, l}^{*}$ are the number of paths and the complex conjugate of the channel coefficient for user $u$. Also, $L_{r}$ and $\alpha_{u, r}^{*}$ are the number of paths and the complex conjugate of the channel coefficient for user $r$, respectively. The Gaussian approximation is used in this paper for deriving the analytic BER of the MU OFDM-OCVSK system. Hence, we can express the decision variable MU OFDMOCVSK system as:

$$
D_{i, u}=\sqrt{P_{0} P_{i}} \frac{d_{i, u} M}{\sum_{m=1}^{M+N_{p} P_{m}}} \sum_{l=1}^{L_{u}} \sum_{v=1}^{\chi_{u}}\left|\alpha_{u, l, v}\right|^{2} E_{b}+B+C+D, i=1, \ldots, M
$$

where $\chi_{u}$ is the number of various channel coefficients through the MU-OFDM-DCSK symbol of user $u$. We can express the mean of the decision variable as:

$$
E\left(D_{i, u}\right)=\sqrt{P_{0} P_{i}} \frac{S_{i, p} M}{\sum_{m=1}^{M+N_{p}} P_{m}} \sum_{l=1}^{L_{u}} \sum_{v=1}^{\chi_{u}}\left|\alpha_{u, l, v}\right|^{2} E_{b}
$$

Because of all the terms in (11) are independent and uncorrelated, assumed the sending bit is +1 , the conditional variance for an arbitrary $i^{\text {th }}$ bit of the decision variable is:

$$
V\left[D_{i}\right]=V[B]+V[C]+V[D]
$$

where $V[$.$] indicates variance, the terms N_{s}, N_{P}^{*}$ and $x_{k, p}^{\prime}$ are independent and uncorrelated, and the channel coefficients are also independent with zero mean variables. The conditional variance of the expression $\mathrm{B}$ can be written as:

$$
V[B]=\left(P_{0}+P_{i}\right) \frac{E_{b} M}{\sum_{m=1}^{M+N p} P_{m}} \frac{N_{0}}{2} \sum_{l=1}^{L_{u}} \sum_{v=1}^{\chi_{u}}\left|\alpha_{p, l, v}\right|^{2}
$$

Likewise, the variance of $\mathrm{C}$ can be:

$$
V[C]=\frac{\beta N_{0}^{2}}{4}
$$

At last, the conditional variance of $\mathrm{D}$ can be written as: 


$$
V[D]=P_{i} \frac{E_{b} M N_{0}}{2 \sum_{m=1}^{M+N_{p}} P_{m}} \sum_{\substack{r=1 \\ r \neq u}}^{U} \sum_{l^{\prime}=1}^{L_{r}} \sum_{v^{\prime}=1}^{\chi_{r}} E\left(\left|\alpha_{u, l^{\prime}, v^{\prime}}\right|^{2}\right)
$$

The value of $E_{B}$ can be considered invariable and the output of the correlator considers as Gaussian random variable. By using the Gaussian approximation, the BER of the $u^{\text {th }}$ user can be calculated as [16]:

$$
\begin{aligned}
B E R & =\frac{1}{2} \operatorname{Pr}\left(D_{i, u}<0 \mid d_{i, u}=+1\right)+\frac{1}{2} \operatorname{Pr}\left(D_{i, u}>0 \mid d_{i, u}=-1\right) \\
& =\frac{1}{2} \operatorname{erfc}\left(\left[\frac{2 V\left(D_{i, u} \mid d_{i, u}=+1\right)}{E\left(D_{i, u} \mid d_{i, u}=+1\right)^{2}}\right]^{-\frac{1}{2}}\right)
\end{aligned}
$$

where $\operatorname{erfc}(\cdot)$ is the complementary error function can be expressed as $\operatorname{erfc}(x)=\frac{2}{\sqrt{\pi}} \int_{x}^{\infty} e^{-t^{2}} d t$ [12]. By using above expressions and (11)-(12), the BER for the MU OFDM-OCVSK system can be written as:

$$
B E R=\frac{1}{2} \operatorname{erfc}\left(\left[\begin{array}{c}
\frac{\left(P_{0}+P_{i}\right)\left(\sum_{m=1}^{M+N p} P_{m}\right) N_{0}}{P_{0} P_{i} M \sum_{l=1}^{L_{u}} \sum_{v=1}^{\chi u}\left|\alpha_{u, l, v}\right|^{2} E_{b}}+ \\
\frac{\beta\left(\sum_{m=1}^{M+N} P_{m}\right)^{2} N_{0}^{2}}{2 P_{0} P_{i}\left(M \sum_{l=1}^{L u} \sum_{v=1}^{\chi u}\left|\alpha_{u, l, v}\right|^{2} E_{b}\right)^{2}}+ \\
\frac{\left(\sum_{m=1}^{M+N} P_{m}\right) N_{0} \sum_{r=1}^{U} \sum_{l^{\prime}=1}^{L_{r}} \sum_{v^{\prime}=1}^{\chi N_{r}} E\left(\left|\alpha_{r, l, v^{\prime}}\right|^{2}\right)}{P_{0} M\left(\sum_{l=1}^{L u} \sum_{v=1}^{\chi u}\left|\alpha_{u, l^{\prime}, v^{\prime}}\right|^{2}\right)^{2} E_{b}}
\end{array}\right]^{-\frac{1}{2}}\right)
$$

From (18), it can be seen that the different strategies of power allocating depending on $P_{0}$, $P_{i}(i=1, . ., M)$ and the total power $\sum_{m=1}^{M+N_{p}} P_{m}$ will produce various values of BER performance. To find optimal values of $P_{m}, m=1, \ldots, M+1$ let define $\Phi(\mathrm{P})$ as:

$$
\Phi(P)=\left[\frac{\left(P_{i}+P_{0}\right) \sum_{m=1}^{M+N p} P_{m} N_{0}}{P_{0} P_{i} M \sum_{l=1}^{L_{u}} \sum_{v=1}^{\chi p}\left|\alpha_{u, l, v}\right|^{2} E_{b}}+\frac{\beta\left(\sum_{m=1}^{M+N_{p}} P_{m}\right)^{2} N_{0}^{2}}{2 P_{0} P_{i}\left(M \sum_{l=1}^{L_{u}} \sum_{v=1}^{\chi_{p}}\left|\alpha_{u, l, v}\right|^{2} E_{b}\right)^{2}}+\frac{N_{0} \sum_{m=1}^{M+N_{p}} P_{m} \sum_{r=1}^{U} \sum_{l^{\prime}=1}^{L_{r}} \sum_{v^{\prime}=1}^{\chi_{r}} E\left(\left|\alpha_{r, l, v^{\prime}}\right|^{2}\right)}{P_{0} M\left(\sum_{l=1}^{L_{u}} \sum_{v=1}^{\chi u}\left|\alpha_{u, l^{\prime}, v^{\prime}}\right|^{2}\right)^{2} E_{b}}\right]
$$

Assume that all subcarriers have the same power, $P_{i}=P$, and normalized the reference power $P_{0}$ to 1 , then $\Phi(P)$ can be rewritten as:

$$
\Phi(P)=\left[\frac{(P+1) \sum_{m=1}^{M+N p} P_{m}}{P M \sum_{l=1}^{L_{u}} \sum_{v=1}^{\chi p}\left|\alpha_{u, l, v}\right|^{2} \frac{E_{b}}{N_{0}}}+\frac{\beta\left(\sum_{m=1}^{M+N p} P_{m}\right)^{2}}{2 P\left(M \sum_{l=1}^{L_{u}} \sum_{v=1}^{\chi p}\left|\alpha_{u, l, v}\right|^{2} \frac{E_{b}}{N_{0}}\right)^{2}}+\frac{\sum_{m=1}^{M+N p} P_{m} \sum_{r \neq 1}^{U} \sum_{l^{\prime}=1}^{L_{r}} \sum_{v^{\prime}=1}^{\chi_{r}} E\left(\left|\alpha_{r, l, v^{\prime}}\right|^{2}\right)}{M\left(\sum_{l=1}^{L_{u}} \sum_{v=1}^{\chi u}\left|\alpha_{u, l^{\prime}, v^{\prime}}\right|^{2}\right)^{2} \frac{E_{b}}{N_{0}}}\right]
$$

Since the $\operatorname{erfc}(\mathrm{x})$ is a monotonous increasing function hence, we consider $\Phi(P)$ is the cost function and the BER will get the optimal value when $\Phi(P)$ approach the minimum value for given $\frac{E_{b}}{N_{0}}, \mathrm{M}$ and spread factor $\beta$. Then the optimization trouble is written as:

Minimize $P_{m}$

Subject to: $\Phi(\mathrm{P}) \leq k$

$$
\begin{aligned}
& \sum_{m=1}^{M} P_{m}+N_{p} \leq P_{T} \\
& P_{m}>0
\end{aligned}
$$

The function $\Phi(\mathrm{P})$ is a posynomial function, therefore a geometric programming (GP) problem [27] with a convex optimization is used to find the minimum value of $\Phi(\mathrm{P})$ utilizing CVX software [28]. For example, when $\frac{E_{b}}{N_{0}}=12 d B$, and $\beta=100$ minimum values of $\Phi(\mathrm{P})$ are $0.2385,0.1174,0.1060$ when $M=24,50$, 64 , respectively, and the corresponding power $P_{m}$ are $0.172403,0.117368,0.106044$.' 


\section{RESULTS AND DISCUSSIONS}

In this section, the analytic and the simulation of BER performance for MU-OFDM-OCVSK proposed system with the subcarriers power allocation strategy are presented. The results are obtained with different lengths of spreading factor $\beta$ and different number of sub-carriers and, number of transmitted bits $\mathrm{M}$, assuming that the number of channel coefficients per OFDM-OCVSK symbol $\chi$ does not change during each frame transmission for different number of users U.The parameters used in the simulations are $\mathrm{M}=(24$, $50,64), \beta=(40,100,150), \mathrm{PT}=(5,10,20)$, with $1,2,3$, and 6 users

Figure 3 shows the BER curves (20) for total number of subcarriers $M=50, \beta=100$, and compares the performance for a 2,3, and 6 users' scenarios under equal power distribution, with that of the optimal power for $\mathrm{PT}=20$. The figure shows that the suggested method with power allocation enhances the performance with each number of users. Further, the effect of the suggested system is more noticed when the number of users is increased. In Figure 4, the impact of subcarriers power allocation on BER performance under different number of subcarriers $M=24$ and 64 and for various number of users $U=1,3$ and 6 users are studied. The spreading factor $\beta$ is set to 100 , and the total power $\mathrm{PT}=20$. Assuming that the bandwidth $B$ is wide enough to support any number of subcarriers $M$. an interesting result of performance improvement is shown in this figure, there is a limited enhancement in the performance when the number of subcarriers is small, as the number of $\mathrm{M}$ is increased, we have a visible performance improvement since more data bits can be sent with the previous number of references.

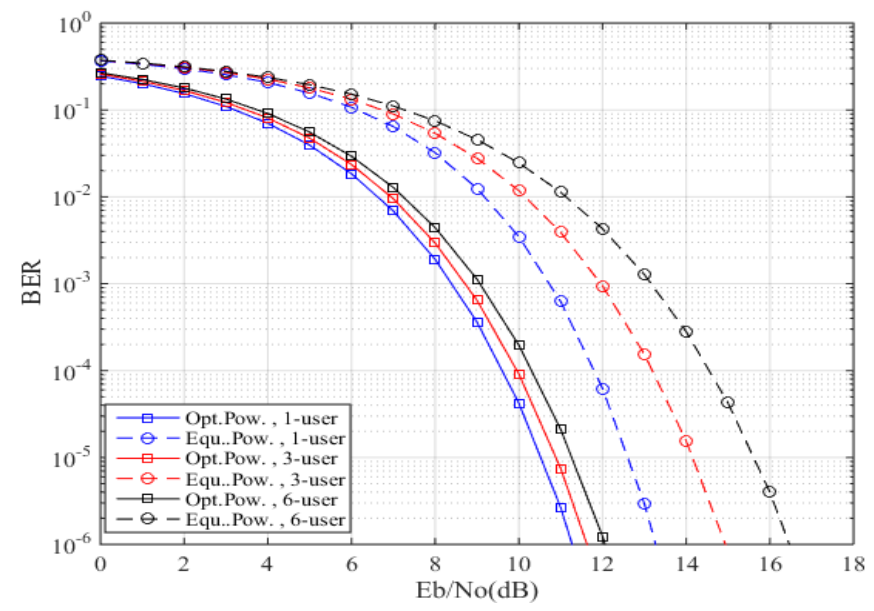

Figure 3. BER performance comparison of the proposed power allocation (opt-pow) with the equal power allocation (Equ. Pow) with $\mathrm{PT}=20, \beta=100, \mathrm{M}=24$, and for different number of users $\mathrm{U}=1,3$, and 6 users

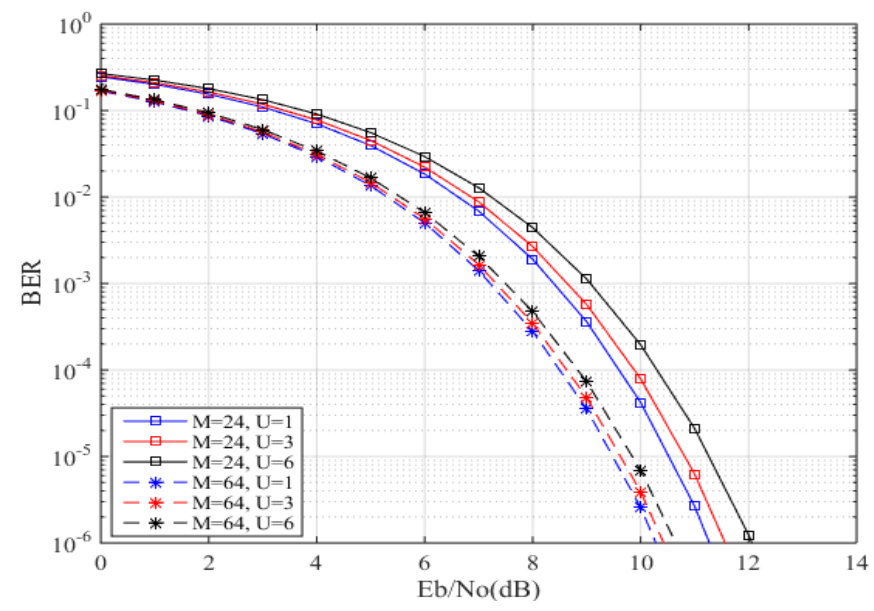

Figure 4. BER performance comparison of the proposed power allocation (opt-pow) with the equal power allocation (Equ. Pow) with PT=20, $\beta=100$, for different number of sub-carriers $M=24,64$ and different number of users $\mathrm{U}=1,3$, and 6 users. 
The effect of the total power $\mathrm{P}_{\mathrm{T}}$ on the performance of the proposed system is presented in Figure 5 . As referred before, the BER expression is a convex function with respect to $\mathrm{P}_{\mathrm{M}}$ and has an inflection point. Thus, it can be seen from the figure that the BER increases as the total power $\mathrm{P}_{\mathrm{T}}$ exceeds a specific value. When $\mathrm{P}_{\mathrm{T}}=10$ we have the lowest BER for $\mathrm{E}_{\mathrm{b}} / \mathrm{N}_{\mathrm{o}}=12 \mathrm{~dB}, \beta=40, \mathrm{M}=24$, and $\mathrm{U}=3$ users.

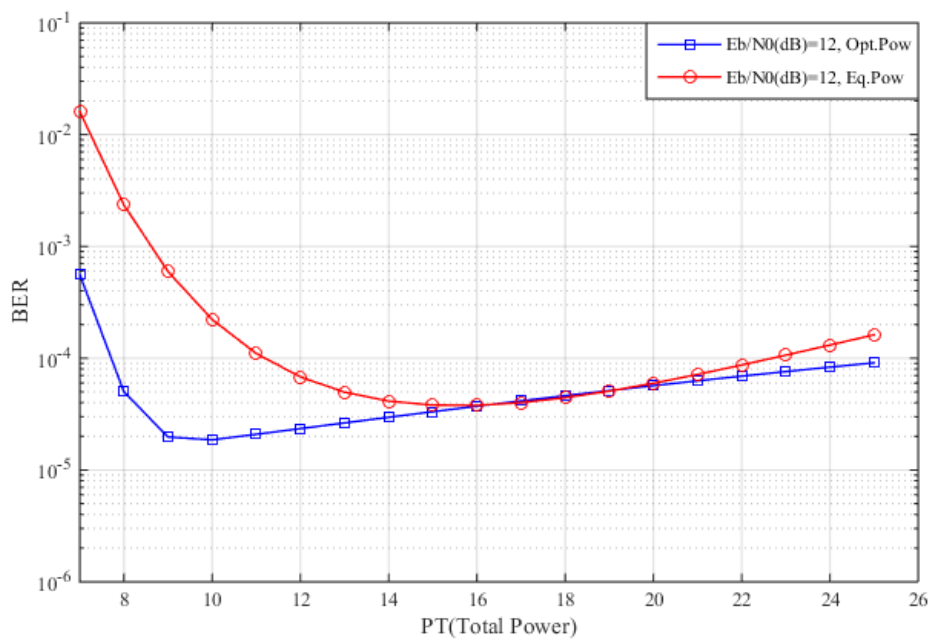

Figure 5. 3-users BER vs. total power $\mathrm{P}_{\mathrm{T}}$ for $\mathrm{E}_{\mathrm{b}} / \mathrm{N}_{\mathrm{o}}=12 \mathrm{~dB}, \beta=40$ and $\mathrm{M}=24$

In Figure 6, BER performance curves of the proposed method and traditional MU-OFDM-DCSK system [19] and MU-OFDM based orthogonal chaotic vector shift keying MU-OFDM-OCVSK system are compared in multipath Rayleigh fading channel with $\mathrm{P}_{T}=20, M=50, \beta=150, \mathrm{~L}_{\mathrm{u}}=3$ and for a 3 users scenario. The proposed optimal power strategy outperforms the conventional MU-OFDM-DCSK and MU-OFDM base orthogonal chaotic vector shift keying MU-OFDM-OCVSK system because of assigning more power to the reference and information bearing subcarriers that make the proposed strategy has a clear performance superiority in terms of BER.

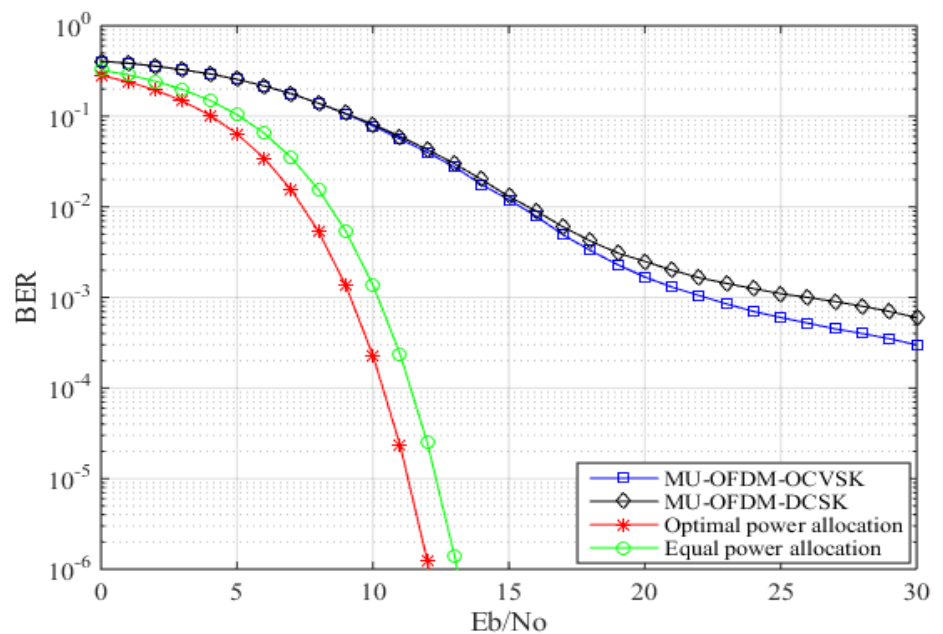

Figure 6. BER performance comparison between the proposed system, MU-OFDM-OCVSK and, MU-

OFDM-DCSK [19] systems when $\mathrm{P}_{\mathrm{T}}=20, \mathrm{M} 50, \beta=150$ and $\mathrm{U}=3$ users

\section{CONCLUSION}

In this paper, the power allocation on subcarriers of MU-OFDM-OCVSK system over multipath frequency selective fading channel is studied. Gaussian approximation is used to derive the BER expression 
and optimal power allocated on subcarriers is studied by convex optimization. The simulation results show that the BER performance of the optimal power allocation technique is enhanced by increasing the number of subcarriers. The results show also that the proper choosing of the total power value improves the BER performance of the system. The BER performance of the proposed optimal power allocation MU-OFDMOCVSK system is plotted and compared with that of traditional MU-OFDM-DCSK and MU-OFDMOCVSK system. The results show an improvement in the performance of the suggested system in terms of bit error rate compared to competitor systems.

\section{REFERENCES}

[1] W. Xu, L. Wang and G. Chen, "Performance of DCSK cooperative communication systems over multipath fading channels," IEEE Transactions on Circuits and Systems I: Regular Papers, vol. 58, no. 1, pp. 196-204, Jan. 2011.

[2] G. Kaddoum and F. Shokraneh, "Analog network coding for multi-user multi-carrier differential chaos shift keying communication system," IEEE Transactions on Wireless Communications, vol. 14, no. 3, pp. 1492-1505, 2015.

[3] M. Dawa, G. Kaddoum and Z. Sattar, "A generalized lower bound on the bit error rate of DCSK systems over multi-path rayleigh fading channels," IEEE Transactions on Circuits and Systems II: Express Briefs, vol. 65, no. 3, pp. 321-325, March 2018.

[4] H. Yang and G. Jiang, "High-efficiency differential-chaos-shift-keying scheme for chaos-based noncoherent communication," IEEE Transactions on Circuits and Systems II: Express Briefs, vol. 59, no. 5, pp. 312-316, 2012.

[5] S. Wang and X. Wang, "M-DCSK-based chaotic communications in MIMO multipath channels with no channel state information," IEEE Transactions on Circuits and Systems II: Express Briefs, vol. 57, no. 12, pp. 1001-1005, Dec. 2010.

[6] P. Chen, L. Wang and G. Chen, "DDCSK-walsh coding: A reliable chaotic modulation-based transmission technique," IEEE Transactions on Circuits and Systems II: Express Briefs, vol. 59, no. 2, pp. 128-132, Feb. 2012.

[7] H. Yang and G. Jiang, "Reference-modulated DCSK: A novel chaotic communication scheme," IEEE Transactions on Circuits and Systems II: Express Briefs, vol. 60, no. 4, pp. 232-236, April 2013.

[8] W. K. Xu, L. Wang, G. Kolumbán, "A novel differential chaos shift keying scheme," International Journal of Bifurcation and Chaos, vol. 21, no. 3, pp. 799-814, 2011.

[9] G. Kaddoum and F. Gagnon, "Design of a high-data-rate differential chaos-shift keying system," IEEE Transactions on Circuits and Systems II: Express Briefs, vol. 59, no. 7, pp. 448-452, July 2012.

[10] F. S. Hasan, "Design and analysis of an orthogonal chaotic vectors based differential chaos shift keying communication system,” Al-Nahrain Journal for Engineering Sciences, vol. 20, no. 4, pp. 952-958, 2017.

[11] W. Xu, Y. Tan, F. C. M. Lau and G. Kolumbán, "Design and optimization of differential chaos shift keying scheme with code index modulation," IEEE Transactions on Communications, vol. 66, no. 5, pp. 1970-1980, May 2018.

[12] G. Kaddoum, F. Richardson and F. Gagnon, "Design and analysis of a multi-carrier differential chaos shift keying communication system," IEEE Transactions on Communications, vol. 61, no. 8, pp. 3281-3291, August 2013.

[13] G. Kaddoum, F. Gagnon and F. Richardson, "Design of a secure multi-carrier DCSK system," 2012 International Symposium on Wireless Communication Systems (ISWCS), pp. 964-968, 2012.

[14] P. Manhas, M. K Soni, "OFDM performance evaluation under different fading channels using MATLAB Simulink," Indonesian Journal of Electrical Engineering and Computer Science, vol. 5, no. 2, pp. 260-266, 2017.

[15] H. Al Ibraheemi and M. A. A. Al Ibraheemi, "Wireless communication system with frequency selective channel OFDM modulation technique," TELKOMNIKA Telecommunication, Computing, Electronics and Control, vol. 18, no. 3, pp. 1203-1208, 2020.

[16] S. Li, Y. Zhao, and Z. Wu, "Design and analysis of an OFDM-based differential chaos shift keying communication system," Journal of Communications, vol. 10, no. 3, pp. 199-205, 2015.

[17] F. S. Hasan and A. A. Valenzuela, "Design and analysis of an OFDM-based orthogonal chaotic vector shift keying communication system," IEEE Access, vol. 6, pp. 46322-46333, 2018.

[18] Z. Liu, L. Zhang, Z. Wu and J. Bian, "Carrier interferometry code index modulation aided OFDM-based DCSK communications," 2019 IEEE 90th Vehicular Technology Conference (VTC2019-Fall), pp. 1-5, 2019.

[19] G. Kaddoum, "Design and performance analysis of a multiuser OFDM based differential chaos shift keying communication system," IEEE Transactions on Communications, vol. 64, no. 1, pp. 249-260, Jan. 2016.

[20] C. Wang, L. Hao, B. Wang, J. Zhang and Y. Lei, "Performance analysis for nonlinear MU-OFDM-DCSK communication systems," 2019 IEEE 10th Annual Ubiquitous Computing, Electronics \& Mobile Communication Conference (UEMCON), pp. 1010-1014, 2019.

[21] N. Zhao, F. R. Yu, H. Sun and M. Li, "Adaptive power allocation schemes for spectrum sharing in interferencealignment-based cognitive radio networks," IEEE Transactions on Vehicular Technology, vol. 65, no. 5, pp. 37003714, May 2016.

[22] M. A. S. Al-Adwany, "Efficient power allocation method for non orthogonal multiple access 5G systems," Indonesian Journal of Electrical Engineering and Computer Science, vol. 10, no. 2, pp. 2139-2150, 2020.

[23] Swapna P. S., Sakuntala S Pillai, Sreeni K. G., "Resource allocation algorithm for symmetrical services in OFDMA systems," Indonesian Journal of Electrical Engineering and Computer Science, vol. 18, no. 2, pp. 867-874, 2020.

[24] W. Xu, L. Wang and T. Huang, "Optimal power allocation in MC-DCSK communication system," 2014 14th International Symposium on Communications and Information Technologies (ISCIT), pp. 313-317, 2014.

[25] J. Bao, W. Xu, L. Wang and T. Huang, "Performance analysis and sub-carriers power allocation for MC-QCSK," 2015 International Conference on Wireless Communications \& Signal Processing (WCSP), pp. 1-5, 2015. 
[26] M. Mobini and M. R. Zahabi, "Power allocation for multi-user OFDM-DCSK system in frequency selective fading channel," Physical Communication, vol. 24, pp. 146-153, 2017.

[27] B. Vandenberghe and L. Vandenberghe, "Convex optimization," Cambridge University Press, pp. 199-200, 2004.

[28] CVX, "MATLAB software for disciplined convex programming," [Online]. Available at. https://cvxr.com/cvx/.

\section{BIOGRAPHIES OF AUTHORS}

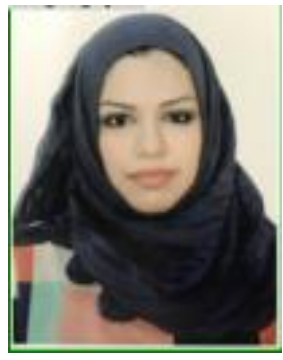

Ansam M. Abed was born in Baghdad, Iraq. She received her degree in Electrical Engineering in 2009 and her M.Sc. degree in Electronics and Communication Engineering in 2012, both from Al- Mustansiriyah University, Iraq. Currently, she is Pursuing Ph.D. program in Communication Engineering at the department of Electrical Engineering, Al- Mustansiriyah University, from 2017. Her recent research activities are OFDM based DCSK, FPGA and Xilinx System Generator based Communication System. Email: ama_ansam@yahoo.com

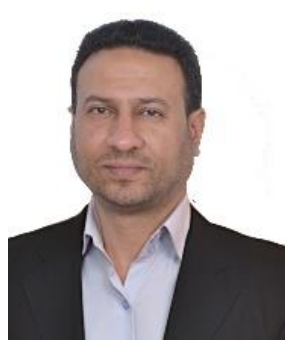

Fadhil S. Hasan was born in Baghdad, Iraq in 1978. He received his B.Sc. degree in Electrical Engineering in 2000 and his M.Sc. degree in Electronics and Communication Engineering in 2003, both from the Mustansiriyah University, Iraq. He received Ph.D. degree in 2013 in Electronics and Communication Engineering from the Basrah University, Iraq. In 2005, he joined the faculty of Engineering at the Mustansiriyah University in Baghdad. His recent research activities are Wireless Communication Systems, Multicarrier System, Wavelet based OFDM, MIMO System, Speech Signal Processing, Image Signal Processing, Cryptography and Computer Security, Chaotic Modulation, FPGA and Xilinx System Generator based Communication System. Now he has been an Assist. Prof. at the Mustansiriyah University, Iraq. Email: fadel_sahib@uomustansiriyah.edu.iq 